\title{
Cytotoxic effect of natural cuttlefish bone xenograft: an in vitro and in vivo study
}

\author{
Komang Agung Irianto, Suyenci Limbong
}

Check for updates

pISSN: 0853-1773 • elSSN: 2252-8083 https://doi.org/10.13181/mji.oa.203141 Med J Indones. 2020;29:136-42

Received: September 15, 2018 Accepted: May 13, 2020

\section{Authors' affiliations:}

Department of Orthopaedic and Traumatology, Faculty of Medicine, Universitas Airlangga, Dr. Soetomo Hospital, Surabaya, Indonesia

\section{Corresponding author:}

Komang Agung Irianto

Department of Orthopaedic and

Traumatology, Dr. Soetomo Hospital,

Jalan Prof. Dr. Moestopo No. 6-8,

Surabaya 60286, Indonesia

Tel/Fax: +62-31-5501481

E-mail: komang168@yahoo.com

\begin{abstract}
BACKGROUND Commercialized synthetic bone grafts are commonly used to replace the bone defect. Cuttlefish bone is naturally available and widely studied, but the specific cytotoxicity test has not been conducted. This study aimed to evaluate the cytotoxicity of the xenograft compared to commercial grafts.
\end{abstract}

METHODS We performed an in vitro test evaluating the viability of human mesenchymal stem cells (hMSCs) when cultured for 48 hours with the tested materials (cuttlefish bone graft and fabricated PerOssal ${ }^{\circledR}$ ). The trypsinized mitochondrial activity of the viable hMSC was assayed based on colorimetry of the formazan color change. The tested material was considered nontoxic if $>70 \%$ of the hMSCs were viable. The in vivo cytotoxic effect was evaluated by implanting the graft material in the femoral muscle of New Zealand (NZ) white rabbits. Nine rabbits were used in each test (cuttlefish bone, PerOssal ${ }^{\circledR}$, and $\left.\mathrm{NaCl} 0.9 \%\right)$. The systemic acute pyrogenic effect was evaluated based on 72 hours body weight changes and rectal temperature changes every 30 min in the first 3 hours and 72 hours post-implantation.

RESULTS The mean percentage of hMSC viability when cultured with cuttlefish bone graft and PerOssal ${ }^{\circledR}$ was comparable ( $93.47 \%$ and $105.37 \%$, respectively, $p=0.240$ ). The in vivo cytotoxicity on NZ rabbit was similar between all tested materials, as shown by the minor changes in body weight ( $<10 \%$ body weight, $p=0.391)$ and rectal temperature $\left(<0.5^{\circ} \mathrm{C}, p=0.127\right)$.

CONCLUSIONS Cuttlefish bone xenograft and fabricated PerOssal ${ }^{\circledR}$ have a similar noncytotoxic effects on hMSCs and non-pyrogenic systemic effects on rabbits.

KEYWORDS bone substitute, cuttlefish bone, cytotoxicity tests, xenograft
Bone grafting is an operative procedure performed to replace a bone defect. ${ }^{1}$ This procedure is the second most frequently performed procedure after blood transfusion.2 According to a previous study, a bone graft may be an autograft, allograft, and xenograft. ${ }^{3}$ Currently, an autograft is still the gold standard for repairing bone defects, as it has osteoconductive, osteoinductive, and osteogenesis properties and provides growth factors with a relatively low risk of infection. ${ }^{1}$ However, bone grafting has several disadvantages, including postoperative pain, hematoma formation, infection, neurovascular trauma, and esthetic deformity, and bone grafts are limited to small defects. ${ }^{4}$ Moreover, allograft and xenograft are developed because of the limited availability of autograft. Allograft requires a 
rigorous preparation to reduce a possible transmission of disease, thus losing most of its osteoconductive, osteoinductive, and osteogenesis capabilities.3,5

Bovine xenograft has been widely used. ${ }^{5,6}$ It plays a role in the development of subsequent xenografts. One of the origins of xenografts is cuttlefish bone; it has a lower production cost, high worldwide availability, and naturally procured. Cuttlefish bone xenograft acts as a scaffold with a high degree of porosity, which is ideal for bone grafting. It has appropriate interconnectivity, suitable for the biologic activity of bone growth, and revascularization. ${ }^{7}$ Following the application of cuttlefish extract, the callus grows thicker with higher osteoblast proliferation, ultimately resulting in better bone healing process. ${ }^{6-8}$ However, before the natural xenograft could be introduced for a human clinical trial, the toxicity of cuttlefish bone xenograft in vitro and in vivo needs to be evaluated to prove the safety of its application.

Synthetic bone grafts are currently used owing to their availability and high reproducibility. ${ }^{3,4}$ The bioavailability of synthetic bone graft is widely investigated, which largely depends on the architectural features of the composite material. ${ }^{1,5}$ PerOssal ${ }^{\circledR}$ is a synthetic bone replacement material that consists of nanocrystalline hydroxyapatite and calcium sulfate. It possesses osteostimulation and osteoinductive properties, which may promote bone healing. In addition, this material may be used to administer antibiotics into a bone defect.

Several studies are evaluating the in vitro and in vivo potency of cuttlefish bone xenograft as a bone replacement or as a scaffold. ${ }^{6-9}$ Nonetheless, before a medical device is allowed for human application, several biocompatibility safety assessments according to U.S. Food and Drug Administration, Japanese guidelines, and International Organization for Standardization should be met. ${ }^{10}$ The in vitro test would be assessed by the viability of human mesenchymal stem cells (hMSCs) after cultured with the tested material. New Zealand (NZ) white rabbit is commonly used as an animal in vivo cytotoxicity test model because of its docility and easy-tomaintain health. ${ }^{11}$ The systemic cytotoxic effect was evaluated based on acute systemic responses such as an alteration in body weight due to diarrhea and loss of appetite and on the pyrogenicity of the implanted material based on rectal temperature changes before and after culture. ${ }^{11,12}$ The rabbit pyrogen test was used for biocompatibility because rabbits respond to pyrogens regardless of their source. ${ }^{11}$ Thus, this study was aimed to compare the in vitro and in vivo toxicity of cuttlefish bone xenograft with a commercially available bone substitute (PerOssal $\left.{ }^{\circledR}\right)$.

\section{METHODS}

This was an experimental study performed at the Laboratory of Tropical Disease Center of Universitas Airlangga, from February through August 2018.

\section{Study design}

The in vitro cell viability test was performed using hMSC cell line incubated for 48 hours in 96-well plates with the tested material. ${ }^{13}$ Cell viability was assayed by colorimetry which measured the optical density (OD) of the cell suspension and compared with the blank well as the control as described by 3-(4,5-dimethylthiazol-2-yl)-2,5-diphenyltetrazolium bromide (MTT) assay. ${ }^{14,15}$ This process was repeated in triplicate.

The in vivo cytotoxic test was a controlled animal laboratory experiment. The minimum number of required samples was calculated using a sample size formula. ${ }^{16}$ In this study, $27 \mathrm{NZ}$ white rabbits (Oryctolagus cuniculus) were divided into three groups, with nine rabbits for each group (cuttlefish bone, PerOssal ${ }^{\circledR}$, and control). This study was fully compliant with animal research on the reporting of in vivo experiments (ARRIVE) criteria. ${ }^{12}$ The study protocol was approved by the Animal Care and Use Committee, Universitas Airlangga, Indonesia (certificate number: 0326/KEPK/V/2018).

\section{Handling of animals}

All rabbits were housed in the animal care laboratory and were well-taken care according to the standards of the National Institute of Health. ${ }^{12}$ The inclusion criteria were as follows: male rabbits, age 6-9 months, healthy without any disability, ambulatory, and body weight of 2,500-3,000 g. The exclusion criteria were surgical wound infection and death. The rabbits were housed individually in separate cages (100 $\times 60 \times 75 \mathrm{~cm}$ ) with following environmental conditions: temperature of $21 \pm 2^{\circ} \mathrm{C}$, humidity of $60 \pm 10 \%$, and lighting of 350 lux intensity with 12-hours dark and light cycle. All rabbits were fed regularly and provided with an unlimited supply of water. 


\section{Preparation of hMSCs}

The human bone marrow was extracted from the femoral shaft of a patient undergoing femoral nailing. The patient has consented to have his/her bone marrow tissue extracted for further research purposes. The bone marrow was put into a 50-ml conical tube, phosphate-buffered saline (PBS) 1:1 was added, suspended to be homogenous, and then carefully layered on $5 \mathrm{ml}$ of Ficoll in a conical tube. It was then centrifuged for $30 \mathrm{~min}$ at $1,600 \mathrm{rpm}$, at $26^{\circ} \mathrm{C}$ until separated into four layers. The second layer was a buffy coat, which looked like a cloudy ring. The buffy coat was put in a $15-\mathrm{ml}$ conical tube, washed with $10 \mathrm{ml}$ of PBS, and centrifuged for $5 \mathrm{~min}$ at 1,600 rpm. The pellet was resuspended in culture media until becoming homogenous, placed in a $10-\mathrm{cm}$ plate, labeled, and then incubated in a $\mathrm{CO}_{2}$ incubator. ${ }^{13}$

\section{Preparation of cuttlefish bone xenograft}

Aragonite $\left(\mathrm{CaCO}_{3}\right)$ was obtained from the lamellae of cuttlefish bone powder, using high-energy milling three-dimensional motion or mortar. It was heated in a furnace at $350^{\circ} \mathrm{C}$ for 3 hours. The $\mathrm{CaCO}_{3}$ content was quantified using X-ray diffraction (XRD). Then, $100 \mathrm{~g}$ of $\mathrm{CaCO}_{3}$ was added to 1 liter of distilled water to acquire a $1 \mathrm{M}$ solution. The $0.6 \mathrm{M} \mathrm{NH}_{4} \mathrm{H}_{2} \mathrm{PO}_{4}$ solution was prepared by dissolving $69 \mathrm{~g}$ of the compound in 1 liter of distilled water., ${ }^{7,16}$ The hydrothermal reaction to produce hydroxyapatite ( $\mathrm{HA})$ was as follows: $10 \mathrm{CaCO}_{3}$ $+6 \mathrm{NH}_{4} \mathrm{H}_{2} \mathrm{PO}_{4}+2 \mathrm{H}_{2} \mathrm{O} \rightarrow \mathrm{Ca}_{10}\left(\mathrm{PO}_{4}\right) 6(\mathrm{OH})_{2}+3\left(\mathrm{NH}_{4}\right)_{2} \mathrm{CO}_{3}$ $+7 \mathrm{H}_{2} \mathrm{CO}_{3}$. The reaction was carried out by mixing two

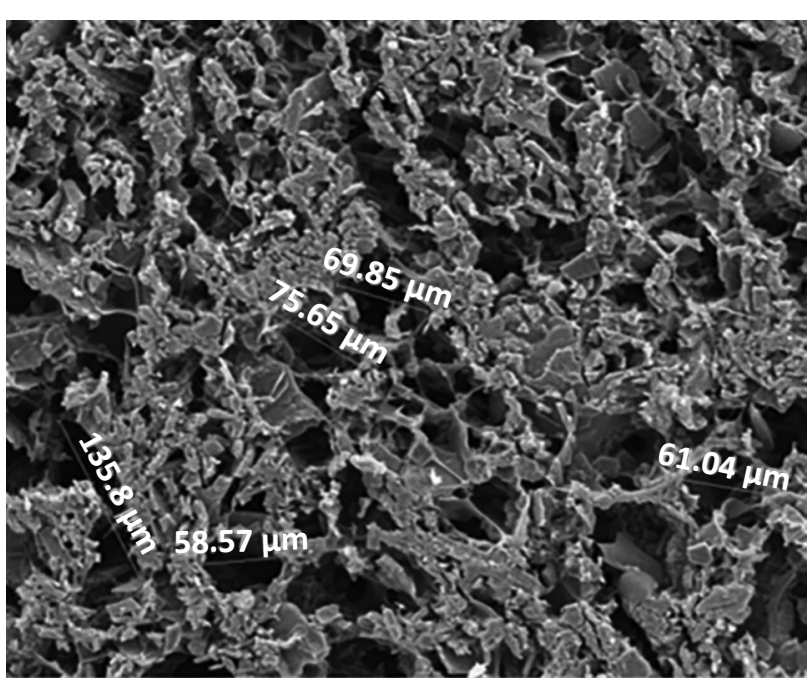

Figure 1. Crystal structure of the cuttlefish hydroxyapatite under the scanning electron microscope previous solutions using a magnetic stirrer for $30 \mathrm{~min}$. The mixture was then heated at $200^{\circ} \mathrm{C}$ for 12 hours. The mixture was then cooled at room temperature and washed with a distilled water using a magnetic stirrer until it was separated from the water and a neutral $\mathrm{pH}$ was achieved. The final washing process contained methanol to limit the agglomeration of HA during the drying process. ${ }^{17}$ The washed sample was filtered through a filter paper and dried in an electric oven at $50^{\circ} \mathrm{C}$ until completely dry. The HA sample that was formed during this process was labeled. The crystal structures were characterized using XRD (Figure 1). The morphology and the ratio $\mathrm{Ca} / \mathrm{PO}_{4}$ were quantified using scanning electron microscope-energy dispersive X-ray spectroscopy.7,17

The synthetic bone graft was PerOssal® (AAP biomaterials $\mathrm{GmbH}$, Germany; product number: 42000000-AKS-004699091). It is in powder form and ready to use. Several other similar products are available from different brands. However, this product is already approved by the Indonesian Food and Drug Regulator (registration number of AKL 21302802278).

\section{Surgical procedure and animal sample preparation}

The animals were anesthetized with ketamine (40 mg/kg) and xylazine (5 mg/kg) intramuscularly and placed prone on a warm pad. The right thigh was disinfected and draped aseptically. The right quadriceps muscle of the control group was injected with $\mathrm{NaCl} 0.9 \%$ alone $(0.5 \mathrm{cc}$ ), while the cuttlefish bone group was injected with cuttlefish bone extract $+\mathrm{NaCl} 0.9 \%$ mixture $(0.5 \mathrm{cc})$, and the Perossal ${ }^{\circledR}$ group was injected with Perossal ${ }^{\circledR}+\mathrm{NaCl} 0.9 \%$ mixture. The bone graft material was injected in the muscle to introduce the possible clinical cytotoxic effect (rectal temperature and body weight changes to represent the health status of the animal sample) as shown in Figure 2. Given the aseptic process, within 72 hours of evaluation, the injection site should not show an inflammatory reaction and functional lesions. If a wound infection is present, the sample will be excluded.

A reduction in body weight is one of the indicators of systemic health status in response to cytotoxicity, which usually appears with diarrhea or loss of appetite. ${ }^{12}$ Body weight was measured before implantation and at 72 hours after implantation. Rectal temperatures may signify immunological systemic reaction in the in vivo models. ${ }^{12}$ The rabbit

mji.ui.ac.id 

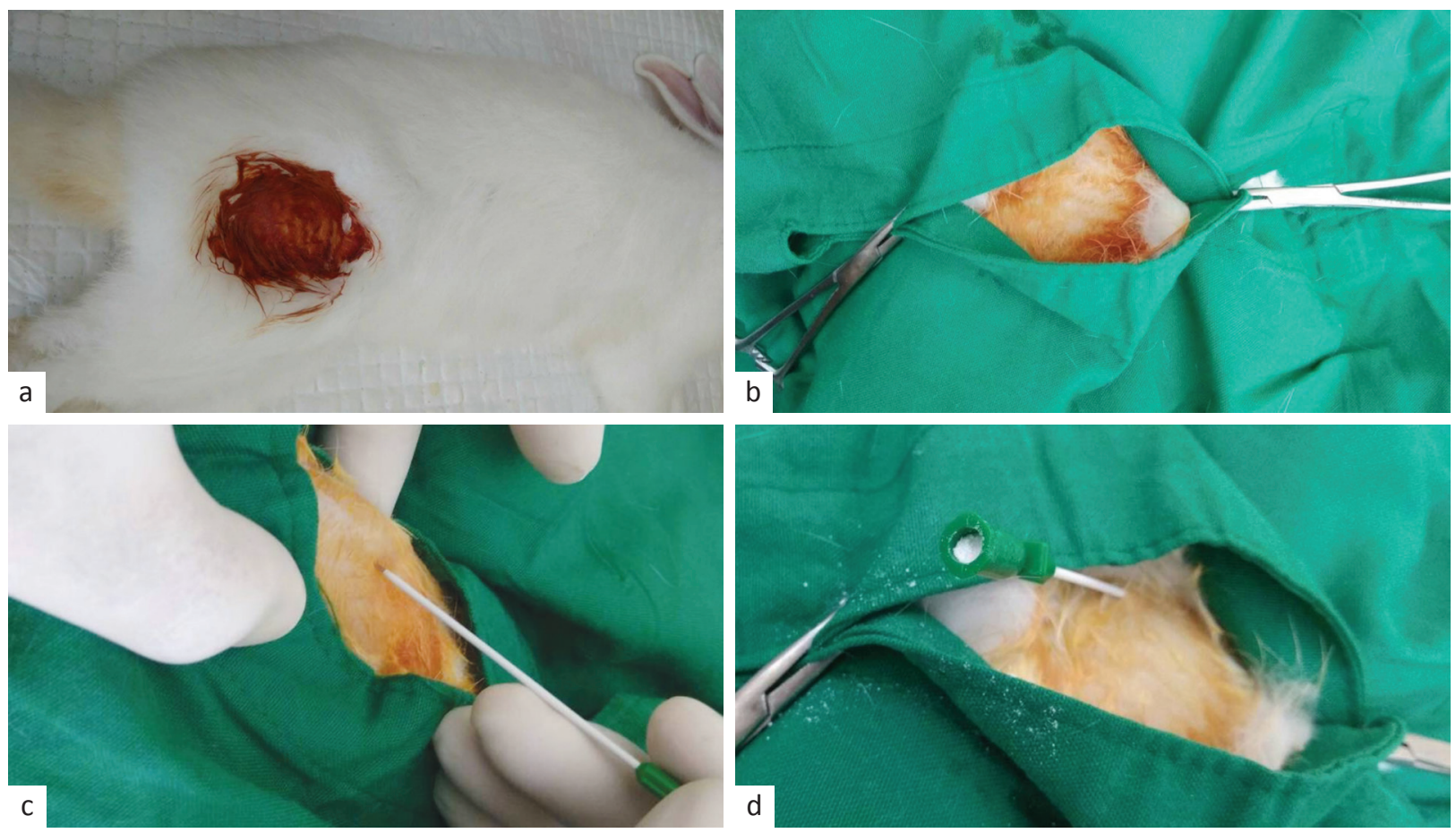

Figure 2. Surgical procedure (sequential clockwise from the upper left). (a) Disinfection; (b) draping; and (c and d) injection of the mixtures ( $\mathrm{NaCl} 0.9 \%$ for the control group, cuttlefish bone extract $+\mathrm{NaCl}$ mixture for the cuttlefish bone group, and PerOssal ${ }^{\circledR}+$ $\mathrm{NaCl}$ mixture for the PerOssal® group)

pyrogenicity test was conducted by recording the rectal temperatures at baseline $(30 \mathrm{~min}$ before injection) and 30-min intervals up to 3 hours postimplantation and 72 hours there after. The tested material is not pyrogenic if no rabbit showed an individual rise in temperature of $\geq 0.5^{\circ} \mathrm{C}$ above its respective control temperature. The requirements for the absence of pyrogenicity are met if not more than three of the nine rabbits have an individual rise in temperature of $\geq 0.5^{\circ} \mathrm{C}$ or if the sum of the maximum rise in temperature of the nine rabbits does not exceed $3.3^{\circ} \mathrm{C} .{ }^{10-12}$ During housing, the health status of the animals were monitored three times daily. No adverse events should be observed in any of the animals. ${ }^{12}$

\section{Cytotoxicity test using MTT assay}

The viability of hMSCs in vitro was evaluated by MTT assay methods on each group. Cell viability was measured based on formazan color change. The cells at $80 \%$ confluent were trypsinized and plated in 96 -well culture plates $\left(1 \times 10^{4}\right.$ cells/well). Each well contained $100 \mu \mathrm{l}$ of cell suspension, and the plates were incubated for 24 hours at $37^{\circ} \mathrm{C}$ in a $5 \% \mathrm{CO}_{2}$ environment. The media was removed from each well after 24 hours. Subsequently, $100 \mu \mathrm{l}$ of eluent from the $0.5,1,5,25,50,100$, or $200 \mu \mathrm{g} / \mathrm{ml}$ cuttlefish bone powder, PerOssal ${ }^{\circledR}$ powder, positive/negative control, and blank wells was placed into the 96-well culture plates $(3 \times 8$ wells/test material), as shown in Figure 3.

Cell viability was assessed after the incubation. The experiments were repeated three times. Then, $100 \%$ viability was determined by the mean OD of the control group (hMSC with media). The OD of the blank well was considered $0 \%$ cell viable. The results for the experimental, positive, and negative control groups were normalized to the blank. The relative cell count ratio was calculated using the following formula (Equation 1): ${ }^{13}$

$\%$ Cell viability $=\frac{\text { optical density sample }- \text { optical density blank }}{\text { optical density control }- \text { optical density blank }} \times 100 \%$

\section{Statistical analysis}

Acquired data were tested by normality test using the Shapiro-Wilk test. If the data were distributed normally, the one-way analysis of variance was used. Otherwise, nonparametric Mann-Whitney test was performed. For repeated measurements, we used the general linear model. If data were not distributed normally, Kruskal-Wallis test was performed. All 
Figure 3. Flow chart of the cytotoxic test using hMSCs and MTT assay. hMSCs=human mesenchymal stem cells; $M T T=3-(4,5$-dimethylthiazol-2-yl)-2,5diphenyltetrazolium bromide

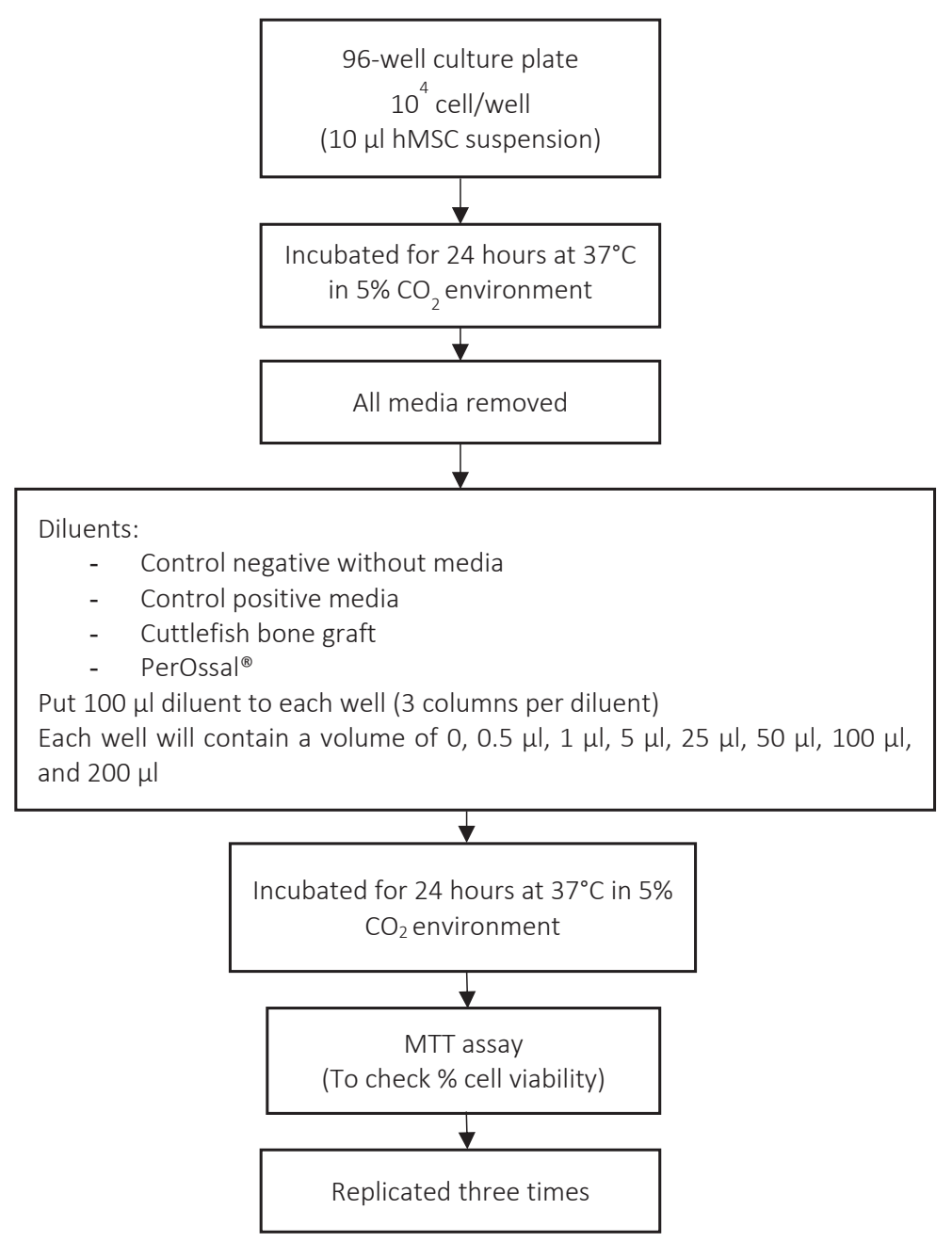

performed. The average rectal temperatures were done after implantation taken at 30-min interval for the first 3 hours and 72 hours. The three tested implant materials showed similar rectal temperature changes before and after the implantation. Since the temperature changes were within the range of $0.5^{\circ} \mathrm{C}$, all cuttlefish bone, PerOssal ${ }^{\circledR}$, and control groups $(\mathrm{NaCl} 0.9 \%)$ passed the pyrogenicity test $(p=0.127)$ (Table 1).

\section{DISCUSSION} deviation) cell viability: cuttlefish bone at 0.9347 $( \pm 0.09)$ and PerOssal $^{\circledR}$ at $1.0573( \pm 0.32) ; p=0.240$ as shown in Figure 4.

Post-hoc analysis using Bonferroni indicated that cuttlefish bone and PerOssal ${ }^{\circledR}$ bone grafts endured similar acute systemic outcome $(p=0.864)$. Body weights shifted less than $5 \%$ (100 g of 2,500 g).

The average rectal temperature of the nine rabbits before implantation was within the healthy normal range. Thus, the pyrogenicity test may be
In vivo cytotoxic test was conducted to ensure the safety of the implant for human application. The short-term in vitro study is not comparable to an in vivo study since most measurements of the toxic effects was only conducted within 12-24 hours after exposure to a toxic substance..$^{14,18}$ In vivo reactions tend to be more complex and possibly performed beyond 24 hours. Thus, it is imperative to use animal samples to test bonegrafts before human clinical 
Figure 4. Human mesenchymal stem cell (hMSC) viability test using cuttlefish bone and PerOssal ${ }^{\circledR}$ materials

*The viability of the cells was quantified based on optical dencity of the cell solution by colorimetry assay and the reference was normal control as $100 \%$, hence it may be exceeded $100 \%$

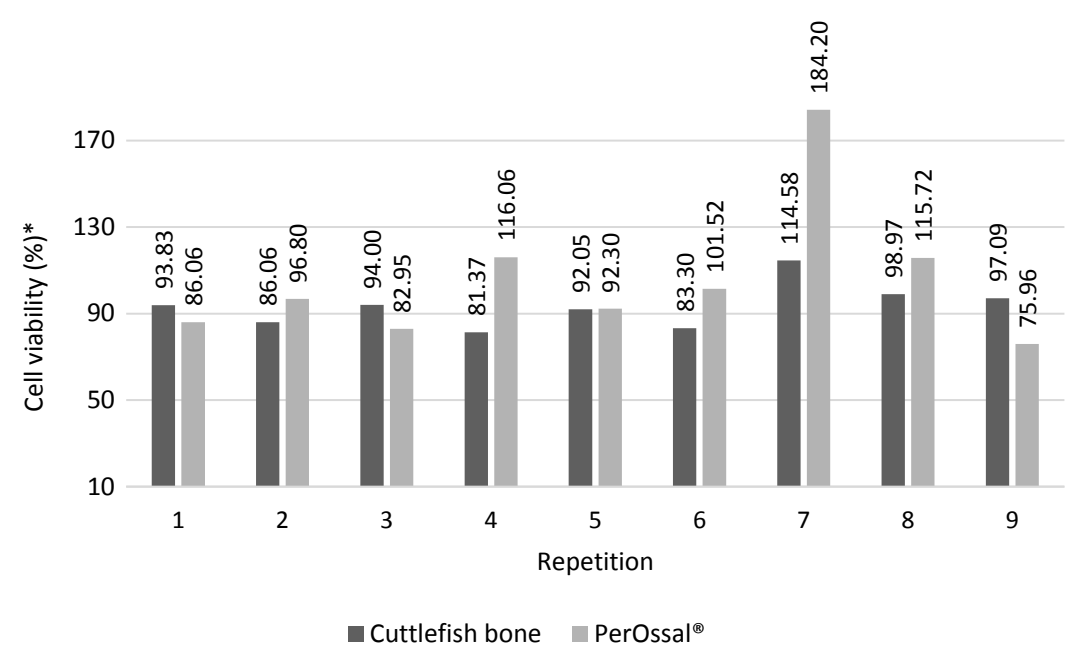

Table 1. Toxicity effect of cuttlefish bone and PerOssal ${ }^{\circledR}$

\begin{tabular}{lcccc}
\hline & In vitro hMSC & \multicolumn{2}{c}{ In vivo (9 NZ rabbits per test)* } \\
\cline { 2 - 5 } & $\begin{array}{c}\text { Cell viability (\%), } \\
\text { mean (SD) }\end{array}$ & $\begin{array}{c}\text { Body weight (g), } \\
\text { mean (SD) }\end{array}$ & $\begin{array}{c}\text { Temperature before intervention }\left({ }^{\circ} \mathrm{C}\right), \\
\text { mean (SD) }\end{array}$ & $\begin{array}{c}\text { Temperature after intervention }\left({ }^{\circ} \mathrm{C}\right), \\
\text { mean }(\mathrm{SD})\end{array}$ \\
\hline Control & 100 & $46.67(10.00)$ & $39.55(0.31)$ & $39.46(0.25)$ \\
PerOssal $^{\circledR}$ & $105.73(32)$ & $45.55(11.30)$ & $39.36(0.35)$ & $39.21(0.29)$ \\
Cuttlefish & $93.47(9)$ & $40.00(11.18)$ & $39.48(0.86)$ & $39.43(0.17)$ \\
$p$ & $0.240^{+}$ & 0.391 & $0.488^{\ddagger}$ & $0.127^{\ddagger}$ \\
\hline
\end{tabular}

hMSC=human mesenchymal stem cell; NZ=New Zealand; SD=standard deviation

*Every rabbit weighted 2,500-3,000 g; ${ }^{\dagger}$ Kruskal-Wallis test; ${ }^{\ddagger}$ one-way analysis of variance

trials. ${ }^{8}$ The animal selection criteria require using animals with similar physiology and pathology with humans. ${ }^{9,11,12}$ In this study, all NZ white rabbits survived until the end of the study, which is in accordance with the nature of NZ white rabbit."

In this study, cuttlefish bonegraft as xenograft has similar cytotoxicity with PerOssal ${ }^{\circledR}$ when incubated with hMSC from human femoral bone marrow. A similar result using osteoblast cell line from rat calvaria was reported by Vajrabhaya et $\mathrm{al}^{14}$ where the decline in viability percentage of the cell was similar in the cuttlefish bone powder group and control group. In this study, the proliferative effect of the cuttlefish bone also showed good results. This fact may be explained by the properties of HA from cuttlefish bone that is quite similar to those found in calcium-based tissues in humans. ${ }^{19}$ Other studies found that the cuttlefish bonegraft has the potency to induce osteoblast differentiation and proliferation. ${ }^{7,20,21}$ This circumstance is postulated because of the presence of $\mathrm{Mg}^{2+}$ on the material, which was proven to promote osteogenesis. ${ }^{22,23}$ Thus, cuttlefish bone powder poses low toxicity and ability to promote osteogenesis, prompting its possible application as bone graft material. ${ }^{14,24}$

The cytotoxic effect in vivo in laboratory animals may present as diarrhea or severe loss of appetite which are usually related to reduced body weight or even death. The rectal temperature signifies an immunological inflammatory reaction in in vivo models. The acute systemic toxicity examination in this study for PerOssal ${ }^{\circledR}$, cuttlefish bone, and control groups showed minor acute systemic toxicity reaction and pyrogenicity, which is consistent with the previous experiment with rat fracture model. ${ }^{6,7,19}$

Dogan and Okumus $^{8}$ reported that cuttlefish bone xenograft did not affect the physiological measurement of the NZ rabbit after 24 weeks of implantation, allowing the cuttlefish bone to become bone xenograft potential material. They also showed no increase in inflammatory cells histologically; thus, the graft does not cause inflammatory reactions..$^{20,22}$ This is in agreement to the implantation of cuttlefish bone extract into Rattus norvegicus with fractured tibial bone that resulted in thicker callus formation and higher proliferation of osteoblast without 
significant inflammatory reaction. ${ }^{6}$ This information signified that an accelerated bone healing process may be affected by the application of cuttlefish bone extract. ${ }^{24,25}$

This study has several limitations. The authors only examine the toxicity effect of cuttlefish bone graft in the acute phase ( 72 hours). The only aspects explored were the pyrogenicity and the effect of the graft to the animals' body weight, and the authors did not evaluate chronic toxicity. Further bioavailability tests need to be conducted to evaluate the effect of cuttlefish bone xenograft in terms of its genotoxicity, sensitization, irritation, chronic toxicity, implant rejection, and carcinogenicity. In conclusion, the cuttlefish bone xenograft has similar nontoxic effect on hMSCs from bone marrow to the fabricated synthetic bone graft PerOssal ${ }^{\circledR}$, and both passed the pyrogenicity rabbit test.

\section{Conflict of Interest}

The authors affirm no conflict of interest in this study.

\section{Acknowledgment}

The authors would like to acknowledge Oen Sindrawati, Yudhistira Pradnyan Kloping, and William PS for editing and revising the final text.

\section{Funding Sources}

None.

\section{REFERENCES}

1. Hung NN. Basic knowledge of bone grafting. In: Zorzi A, editor. Bone grafting. London: IntechOpen; 2012. p. 11-38. Available from: https://www.intechopen.com/books/bone-grafting/basicknowledge-of-bone-grafting.

2. Kinaci A, Neuhaus V, Ring DC. Trends in bone graft use in the United States. Orthopedics. 2014;37(9):e783-8.

3. Finkemeier CG. Bone-grafting and bone-graft substitutes. J Bone Joint Surg Am. 2002;84(3):454-64.

4. Pape HC, Evans A, Kobbe P. Autologous bone graft: properties and techniques. J Orthop Trauma. 2010;24Suppl 1:S36-40.

5. Sarin P, Lee SJ, Apostolov ZD, Kriven WM. Porous biphasic calcium phosphate scaffolds from cuttlefish bone. J Am Ceram Soc. 2011;94(8):2362-70.

6. Kloping LP, Purwati, Edward M. The healing effect of cuttlefish bone on fractured bone in rat model. Bali Med J. 2016;5(2):193-6.

7. Irianto KA, Pribadi A, Irsyam IA, Kloping YP, Sindrawati O. A comparison of osteoblast cell proliferation and osteocalcin expression in cuttlefish bone and bovine bone xenograft. Mol
Cell Biomed Sci. 2019;3(2):75-80.

8. Dogan E, Okumus Z. Cuttlebone used as a bone xenograft in bone healing. Vet Med. 2014;59(5):254-60.

9. Cadman J, Zhou S, Chen Y, Li Q. Cuttlebone: characterisation, application and development of biomimetic materials. J Bionic Eng. 2012;9(3):367-76.

10. Medical Device and Diagnostic Industry (MDDI) Online. Biocompatibility safety assessment of medical devices: FDA, ISO, and Japanese guidelines [Internet]. 2000 [cited 2018 Sep 18]. Available from: https://www.mddionline.com/ biocompatibility-safety-assessment-medical-devices-fdaisoand-japanese-guidelines.

11. Mapara M, Thomas BS, Bhat KM. Rabbit as an animal model for experimental research. Dent Res J. 2012;9(1):111-8.

12. Kilkenny C, Browne WJ, Cuthill IC, Emerson M, Altman DG. Improving bioscience research reporting: the ARRIVE guidelines for reporting animal research. PLoS Biol. 2010;8(6):e1000412.

13. Rantam FA, P Ferdiansyah, A Purwati. Mesenchymal stem cell, hematopoietic, and application model. Surabaya: Universitas Airlangga; 2014. Indonesian.

14. Vajrabhaya LO, Korsuwannawong S, Surarit R. Cytotoxic and the proliferative effect of cuttlefish bone on MC3T3-E1 osteoblast cell line. Eur J Dent. 2017;11(4):503-7.

15. BioVision Incorporated. Ready-to-use cell proliferation colorimetric reagent, WST-1 (catalog \#K304-2500; assays; store at $-20^{\circ} \mathrm{C}$ ). Milpitas: BioVision Incorporated.

16. Supranto J. Simple random sampling. In: Supranto J. Sampling technique in survey and experimental study. Jakarta: Rineka Cipta; 2000. Indonesian.

17. Aminatun, Siswanto, Penga YM, Istifarah, Apsari R. The effect of sintering process on the characteristics of hydroxyapatite from cuttlefish bone (Sepia Sp.). Res J Pharm Biol Chem Sci. 2013;4(4):1431-42.

18. Dimarino AM, Caplan Al, Bonfield TL. Mesenchymal stem cells in tissue repair. Front Immunol. 2013;4:201.

19. Park JW, Bae SR, Suh JY, Lee DH, Kim SH, Kim H, et al. Evaluation of bone healing with eggshell-derived bone graft substitutes in rat calvaria: a pilot study. J Biomed Mater Res A. 2008;87(1):20314.

20. Guda T, Walker JA, Pollot BE, Appleford MR, Oh S, Ong JL, et al. In vivo performance of bilayer hydroxyapatite scaffolds for bone tissue regeneration in the rabbit radius. J Mater Sci Mater Med. 2011;22(3):647-56.

21. Blokhuis TJ, Arts JJ. Bioactive and osteoinductive bone graft substitutes: definitions, facts and myths. Injury. 2011;42 Suppl 2:S26-9.

22. Cerrolaza M, Duarte V, Garzón-Alvarado D. Analysis of bone remodeling under piezoelectricity effects using boundary elements. J Bionic Eng. 2017;14(4):659-71.

23. Checa AG, Cartwright JH, Sánchez-Almazo I, Andrade JP, RuizRaya F. The cuttlefish Sepia officinalis (Sepiidae, Cephalopoda) constructs cuttlebone from a liquid-crystal precursor. Sci Rep. 2015;5:11513.

24. Oryan A, Alidadi S, Moshiri A, Maffulli N. Bone regenerative medicine: classic options, novel strategies, and future directions. J Orthop Surg Res. 2014;9(1):18.

25. Nazirkar G, Singh S, Dole V, Nikam A. Effortless effort in bone regeneration: a review. J Int Oral Health. 2014;6(3):120-4. 